Revue musicale OICRM

\title{
Présentation du numéro « Une relève »
}

\section{Michel Duchesneau}

Volume 2, numéro 2, 2015

Une relève

URI : https://id.erudit.org/iderudit/1060128ar

DOI : https://doi.org/10.7202/1060128ar

Aller au sommaire du numéro

Éditeur(s)

OICRM

ISSN

2368-7061 (numérique)

Découvrir la revue

\section{Citer ce document}

Duchesneau, M. (2015). Présentation du numéro « Une relève ». Revue musicale OICRM, 2(2), i-iii. https://doi.org/10.7202/1060128ar d'utilisation que vous pouvez consulter en ligne.

https://apropos.erudit.org/fr/usagers/politique-dutilisation/ 


\section{revue musicale oicrm}

Le site de la Revue musicale de l'Observatoire interdisciplinaire de création et recherche en musique

\section{Présentation du numéro "Une relève ", vol. 2, n 2 (2015) \\ Michel Duchesneau}

Ce numéro de la Revue musicale OICRM est doublement particulier. D'une part, il est le premier à paraître sous une nouvelle apparence numérique, raffinée et évoluée que l'on doit au remarquable travail d'Anne Lardeux appuyé par l'expertise informatique de Jean-Michel Dumas et de Tiago Bortoletto Vaz et, d'autre part, il est entièrement consacré aux travaux d'étudiants rattachés à l'OICRM. Si l'un des principaux objectifs de la Revue musicale OICRM est de diffuser les travaux réalisés au sein du centre, il semblait essentiel aux membres du comité scientifique

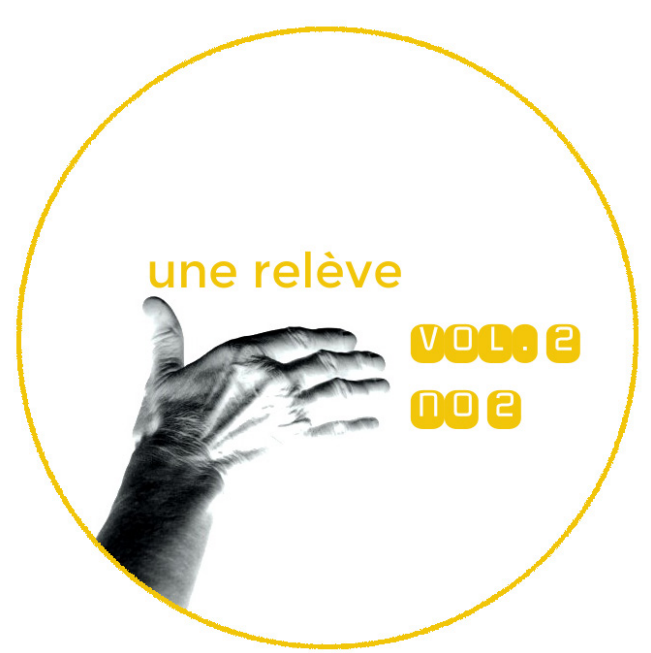
de l'oicRm qui fait office de comité de rédaction d'en témoigner en mettant en lumière la part essentielle des étudiants de maîtrise, de doctorat et des stagiaires postdoctoraux dont les travaux de recherche contribuent largement au rayonnement du centre. C'est, je l'espère, mission accomplie.

L'intérêt de ce numéro est, quant à lui, triple : diversité des sujets, variété des approches et représentativité disciplinaire. Si l'analyse de la musique de cinéma domine avec deux articles, c'est un hasard et il faut porter une attention particulière à l'élargissement des objets de recherche que propose la revue. De la world music à Claude Debussy en passant par la dance music, des questions d'appropriation des matériaux anciens dans les œuvres contemporaines à la musicalisation des effets sonores dans Star Wars, pour ne citer que ces thèmes, on a là un échantillon bouillonnant des travaux des étudiants des cycles supérieurs en cours de réalisation à l'OICRM.

Le présent numéro est aussi un témoignage éloquent de l'évolution des études sur la musique au cours de la dernière décennie. Compte tenu de la nature du numéro, il n'y a pas à proprement parler de fil conducteur qui relierait les articles les uns aux 
autres. Mais chaque article met en relief les intérêts et les approches privilégiées par une nouvelle génération de chercheurs. Les travaux en recherche-création, l'étude des répertoires inédits appartenant à la sphère de l'audiovisuel et les approches interdisciplinaires réellement mise en application, caractérisent le travail de cette nouvelle génération talentueuse.

Ce talent se manifeste évidemment dans la qualité des articles qui ont été évalués par un comité international de pairs. Processus habituel des revues scientifiques. Mais dans notre cas, ces derniers ont assuré un mentorat exceptionnel. Je tiens à les en remercier. Le processus a permis aux auteurs Ons Barnat, Liouba Bouscant, Ariane Couture, Solenn Hellégouarch, Chloé Huvet, Anthony Papavasiliou et Benjamin Lassauzet, grâce à de nombreux conseils des évaluateurs, d'approfondir leur réflexion, alimentant du coup leur article, mais aussi leurs travaux de recherche en cours ou à venir.

La revue comporte désormais une section nommée " Notes de terrain ». Réservée à des essais, des témoignages, des présentations d'expériences en cours, des comptes rendus d'événement, de livres ou d'enregistrement, elle donne la possibilité aux chercheurs qu'ils soient de l'OICRM ou pas, de présenter, sous une forme libre, une réflexion vivante sur la recherche en musique. Nous accueillons pour ce numéro les textes de Véronique Daigle, Fabien Genthialon et Philippe Béland.

ARTICLES

Matériaux anciens dans la musique contemporaine actuelle. Postmodernisme et modernisme en questions

1 Liouba Bouscant

(Re)présenter les œuvres musicales. L'exemple des programmes de concert de la Société de musique contemporaine du Québec

44 Ariane Couture

Musique et effets sonores dans Star Wars : Épisode II - L'attaque des clones. Une alliance conflictuelle?

67 Chloé Huvet

David Cronenberg et Howard Shore. Bref portrait d'une longue collaboration

96 Solenn Hellégouarch

L'analyse des ensembles microrythmiques dans 1'Intelligent Dance Music

115 Anthony Papavassiliou

Hybridité, authenticité et atteinte du succès international ; réflexion sur les processus de commercialisation de disques de world music

133 Ons Barnat 
Debus-si e(s)t Pierrot. Rire pour ne pas pleurer

154 Benjamin Lassauzet

NOTES DE TERRAIN

Quels liens établir entre la philosophie praxialiste de David Elliott et le programme de formation de niveau primaire du Ministère de l'Éducation au Québec (PFEQ) dans le domaine de la musique?

178 Veronique Daigle

Mon regard d'interprète. L'interprétation, un processus indissociable du texte musical

190 Fabien Genthialon

Compte rendu du douzième Forum international des jeunes compositeurs

197 Philippe Béland

Couverture : photo de Lou Scamble.

Graphisme : Anne Lardeux. 